\title{
Microfilaments and Actin-Associated Proteins at Sites of Membrane-Substrate Attachment within Acetylcholine Receptor Clusters
}

\author{
ROBERT J. BLOCH, ${ }^{* 1}$ MARISELA VELEZ, $\dagger$ \\ JACQUELINE G. KRIKORIAN,* and DANIEL AXELROD ${ }^{\dagger}$ \\ *Department of Physiology, University of Maryland School of Medicine, 660 W. Redwood St., \\ Baltimore, Maryland 21201, and †Biophysics Research Laboratory, University of \\ Michigan, 2200 Bonisteel Blvd., Ann Arbor, Michigan 48109
}

\begin{abstract}
Rat myotubes in tissue culture form broad areas of close contact with the substrate. These areas often display two distinct, interdigitating sets of membrane domains. One, the "contact domain", is close to the substrate; the other, termed the "AChR domain", is further from the substrate and is rich in acetylcholine receptors (AChR). We have used fluorescence techniques to study the organization of the cytoskeleton in these areas. Substrate-apposed membrane of the myotubes was exposed either by shearing or by permeabilizing the cells with a neutral detergent. Phalloidin derivatives and affinitypurified polyclonal or monoclonal antibodies specific for cytoskeletal proteins were then applied to the samples. Sheared samples were observed by epifluorescence microscopy; detergent-permeabilized samples were observed by total internal reflection fluorescence microscopy. We found that, like antivinculin, fluorescent phalloidin derivatives and antibodies to $a$-actinin, filamin, and talin preferentially labeled the contact domains. This suggests that bundles of microfilaments associate with the membrane at sites of myotubesubstrate attachment. In contrast, a $43 \mathrm{~K}$ protein, closely associated with $\mathrm{AChR}$, was present only at AChR domains. A monoclonal antibody to actin labeled both AChR and contact domains, suggesting that actin is enriched over both regions. Our results suggest that, like the plasma membrane of AChR clusters, the underlying membrane skeleton is organized into at least two distinct domains. () 1989 Academic Press, Inc.
\end{abstract}

The postsynaptic membrane of the vertebrate neuromuscular junction is highly specialized to bind acetylcholine released by the motor neuron and to convert this binding into a change in membrane potential. The acetylcholine receptor (AChR) is the component of the postsynaptic membrane responsible for these events. At the healthy, adult synapse, AChR is present at very high densities, in paracrystalline arrays at the tips of the secondary postsynaptic folds (for reviews, see Refs. $[1,2] . A C h R$ are present at much lower densities in extrajunctional regions of the muscle. Numerous studies with normal and diseased muscles have shown that high concentrations of $\mathrm{AChR}$ in the postsynaptic membrane are required for efficient neurotransmission at the neuromuscular junction. Consequently, great interest has focused on the mechanism of AChR accumulation in the postsynaptic region of muscle.

The subsynaptic cytoskeleton has been extensively studied to learn how it interacts with the postsynaptic membrane. To date, 10 different cytoskeletal proteins have been found to be enriched at the postsynaptic region, including

${ }^{1}$ To whom reprint requests should be addressed. 
actin and a number of actin-associated proteins [3-6], a tonofilament-like protein [7], and proteins with polypeptide chain molecular weights of 58,000 and 270,000 or $300,000[8,9]$ (see Refs. $[10,11]$ for reviews). The functions of these proteins in generating or maintaining the structure of the postsynaptic region are not known.

Several of the proteins that are enriched in the postsynaptic region of the neuromuscular junction are, in other cells, associated with sites of cell-substrate or cell-cell adhesion. These include vinculin, talin, and $\alpha$-actinin, all proteins present at focal contact sites where bundles of microfilaments associate with the membrane [12-15]. Only vinculin, however, has been localized at sites of cellsubstrate contact in cultured rat muscle cells [16]. The relationship of cytoskeletal proteins to sites of cell-membrane contact in these cells is of interest because it is at these sites that large clusters of AChR form [16-20]. Many of the properties of these substrate-apposed AChR clusters are similar to those of AChR clusters that form at the embryonic neuromuscular junction [11].

The AChR clusters of rat myotubes cultured in vitro are often organized into a series of distinct, interdigitating membrane domains, one rich in $\mathrm{AChR}$, termed the "AChR domain", the other poor in AChR and closer to the substrate, termed the "contact domain" $[16,21]$. This segregation permits one to distinguish between cytoskeletal proteins associated preferentially with sites of cell-substrate attachment, and those associated more closely with AChR. We have used this system to investigate the distribution of some of the actin-binding proteins that are enriched in the postsynaptic region of the neuromuscular junction. We show here that bundles of microfilaments, visualized with fluorescent derivatives of phalloidin, are found preferentially over contact domains, and that these regions are enriched in talin, filamin, and occasionally in $\alpha$-actinin. Like phalloidin, antibodies to actin label contact domains, but, unlike phalloidin, they also label the AChR domains, in agreement with an earlier report [4]. Our results suggest that, like the plasma membrane of AChR clusters, the underlying membrane skeleton is organized into at least two distinct domains.

\section{METHODS}

Cultures of rat myotubes were prepared on glass coverslips, as described in earlier reports from our laboratories [16, 22, 23]. Briefly, neonatal rat hind limb muscle was dissociated with collagenase, and suspended at $10^{6}$ cells $/ \mathrm{ml}$ in Dulbecco-Vogt modified Eagle's medium containing $10 \%$ fetal calf serum (medium). Aliquots $(0.3-0.4 \mathrm{ml})$ were seeded onto $25-\mathrm{mm}$ round glass coverslips. One day later, an additional $1.5 \mathrm{ml}$ medium was added to the cultures. Medium was replaced on Day 4 with fresh medium containing $20 \mu M$ cytosine arabinoside, added to reduce the growth of non-muscle cells. Cells were usually examined 6 to 8 days after plating, when large clusters of AChR had formed in the substrate-apposed membranes of the myotubes. At this stage, myotubes were approximately $400 \mu \mathrm{m}$ long, and ranged in width from 15 to $40 \mu \mathrm{m}$. AChR clusters usually formed at the widest segments of myotubes. Cultures to be sheared and observed by epifluorescence were usually labeled with monotetramethylrhodamine- $\alpha$-bungarotoxin (K-BI) [24]. Cultures to be observed by total internal reflection fluorescence (TIRF) were labeled with fluoresceinated $a$-bungarotoxin (F-BT), prepared from fluorescein isothiocyanate following the same method as used for R-BT [24].

For experiments using TIRF, cells were extracted for $1 \mathrm{~min}$ at ambient temperature with a solution containing $0.5 \%$ Triton X-100 (0.5\% Triton X-100, $200 \mathrm{mM}$ sucrose, $50 \mathrm{mM} \mathrm{NaCl}, 5 \mathrm{mM} \mathrm{MgCl}$, $0.02 \%$ sodium azide, $10 \mathrm{~m} M$ Hepes, $\mathrm{pH} 7.0$ ) [16]. Samples were fixed for $15 \mathrm{~min}$ in ice cold $2 \%$ paraformaldehyde, and treated with $0.1 \mathrm{M}$ glycine in buffered saline, to inactivate any remaining aldehyde. They were then labeled with antibodies, diluted in buffered saline supplemented with $0.1 \%$ 
bovine serum albumin, for $30 \mathrm{~min}$ at room temperature. Samples were washed several times over the next half hour and then counterstained for $1 \mathrm{~h}$ with rhodaminylated goat anti-rabbit (RGAR) or antimouse (RGAM) antibodies (Cappel Laboratories, Organon Technikon, West Chester, PA; used at a final dilution of 1:50). After repeated washing, samples were inverted over a droplet of Hanks' balanced salt solution on a $25 \mathrm{~mm}$ glass coverslip, contained within a $60 \mu \mathrm{m}$ thick, annulus-shaped Teflon spacer. The salt solution was supplemented with an enzyme and substrate to eliminate oxygen, which would otherwise accelerate photobleaching of the fluorophores. In some experiments, this supplement consisted of $1 \mu M$ protocatechoic acid deoxygenase (the gift of Dr. James Fee, Los Alamos National Laboratory) and its substrate, protocatechoic acid. An aliquot of a stock solution of the latter ( $2 \mu$ l of a $0.1 M$ solution in $0.1 M \mathrm{NaOH}$ ) was introduced into the solution bathing the sample immediately before the chamber was sealed. In other experiments, the solution was supplemented with $0.5 \mathrm{mg} / \mathrm{ml} p$-phenyl diamine and taken to $\mathrm{pH} 8.6$ in order to retard the bleaching rate of fluorescein [25]. The samples in the sealed chambers were then observed using a Leitz Diavert inverted microscope equipped with an argon laser for TIRF [18]. Different samples were viewed through one of the following objectives: Leitz 50x water immersion (NA 1.00), Leitz 100x water immersion (NA 1.20), or Zeiss $63 \times$ oil immersion (NA 1.40). The F-BT image was always photographed first, using the 488-nm argon line; exposure was usually for $30 \mathrm{~s}$. Rhodamine fluorescence was photographed for approximately $30 \mathrm{~s}$ under illumination at $514 \mathrm{~nm}$. Exposures were for times long enough to bleach approximately half of the fluorophore in the field. The dichroic mirrors and barrier filters were custom designed for this laser system to reduce spectral overlap to a level considerably lower than that normally achieved with a mercury arc and commercial filter packs for these fluorophores. Kodak TMAX P3200 film was processed to an ASA of 25,000, following the instructions of the manufacturer.

Myotubes were also labeled with antibodies after shearing, which removes most of the cytoplasm overlying the AChR clusters. In early experiments, samples were sheared with a stream of ice-cold $2 \%$ paraformaldehyde in buffered saline [16]. Later, samples were first treated for $2 \mathrm{~min}$ at RT with $\mathrm{ZnCl}_{2}$ (1 $\mathrm{m} M \mathrm{ZnCl}_{2}, 3 \mathrm{~m} M$ EGTA, $5 \mathrm{~m} M \mathrm{MgCl}_{2}, 100 \mathrm{~m} M$ Pipes, $\mathrm{pH}$ 6.0) [26], and then sheared with a stream of ice-cold high potassium buffer $(100 \mathrm{mM} \mathrm{KCl}, 5 \mathrm{mM} \mathrm{MgCl}, 3 \mathrm{mM}$ EGTA, $20 \mathrm{mM}$ Hepes, pH 7.0) [27]. This procedure was adapted for use with myotube cultures by Dr. D. W. Pumplin (Department of Anatomy, University of Maryland School of Medicine), who kindly provided us with the details. It gives a much higher yield of substrate-associated AChR clusters than shearing directly in paraformaldehyde. The results obtained with the two methods were identical. After fixation for 15 min in $2 \%$ paraformaldehyde and treatment with glycine, samples were labeled for $30 \mathrm{~min}$ at room temperature with primary antibody. They were then washed several times over $30 \mathrm{~min}$, counterstained with FGAR or FGAM (Cappel Laboratories, or Jackson Immunoresearch, West Grove, PA; used at $1: 100$ ), and washed again. Samples were mounted in 1 part $1 \mathrm{M}$ Tris- $\mathrm{HCl}, \mathrm{pH} \mathrm{8.0,9}$ parts glycerol, or in the same solution supplemented with $1 \mathrm{mg} / \mathrm{ml} p$-phenylenediamine, to reduce photobleaching [28]. Samples were observed with a Zeiss $63 \times$ PlanNeofluar oil immersion objective (NA 1.25) on a Zeiss IM-35 microscope equipped for epifluorescence. Ilford HP-5 film exposed for 15-45 s was used to photograph sheared clusters. The film was processed with Ilford Microphen to an ASA of $\sim 1600$.

Some sheared samples were also labeled with rhodamine or fluorescein derivatives of phalloidin (Molecular Probes, Eugene, OR), either with or without prior labeling with R-BT or antibodies. In all cases, samples already labeled with R-BT, antibodies, or both were incubated for $10 \mathrm{~min}$ at room temperature with $330 \mathrm{nM}$ phalloidin derivative in buffered saline, washed quickly with buffered saline supplemented with $1 \mathrm{mg} / \mathrm{ml} \mathrm{BSA}$, and then mounted for observation.

Affinity-purified rabbit antibodies to talin were kindly provided by Dr. J. Wilkins (Department of Biophysics, The Johns Hopkins University, Baltimore, MD) and by Dr. K. Burridge (Department of Cell Biology and Anatomy, University of North Carolina, Chapel Hill, NC). Monoclonal antibody 1579A specific for the postsynaptic $43 \mathrm{~K}$ protein was the kind gift of Dr. S. Froehner (Department of Biochemistry, Dartmouth Medical School, Hanover, NH). Other antibodies have been described in earlier reports [4, 5]. The specificities of all the affinity-purified rabbit antibodies were tested by immunoblotting, following the method of Burnett [29], as described [4]. Polyacrylamide gel electrophoresis in the presence of sodium dodecyl sulfate was performed by the method of Laemmli [30]. Antibodies bound to the nitrocellulose blots were detected with radioiodinated protein A ( ${ }^{123}$ I-protein A). Vinculin, $\alpha$-actinin, and filamin were purified as described [31]. Purified talin was the gift of Dr. K. Burridge.

Protein A was purchased from Pharmacia (Piscataway, NJ) and was iodinated using chloramine T. Other chemicals were purchased from Sigma Chemical Co. (St. Louis, MO). Inorganic salts were reagent grade, from J. T. Baker (Phillipsburg, NJ). 


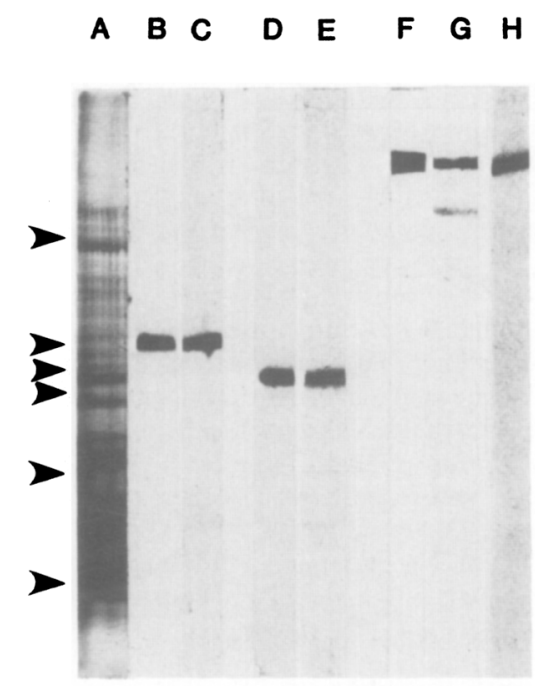

Fig. 1. Immunoblotting analysis of affinity-purified antivinculin, anti- $\alpha$-actinin and antifilamin. Purified proteins and extracts of rat myotubes were subjected to SDS-PAGE on minigels. Samples in lanes $B-H$ were transferred electrophoretically onto nitrocellulose paper. Strips were cut from each lane and incubated with one of the affinity-purified antibodies, followed by ${ }^{125} \mathrm{I}$-protein $\mathrm{A}$. Blots were visualized by autoradiography. (A) Total myotube protein, stained with Coomassie blue. (B) Purified vinculin, labeled with affinity-purified antivinculin. (C) Total myotube protein labeled with affinitypurified antivinculin. The only labeled band coelectrophoreses with vinculin $(D)$ Purified $\alpha$-actinin labeled with affinity-purified anti- $\alpha$-actinin. $(E)$ Total myotube protein labeled with affinity-purified anti- $\alpha$-actinin. Only a band coelectrophoresing with $\alpha$-actinin is labeled. $(F)$ Purified filamin labeled with affinity-purified antifilamin. $(G)$ Total myotube protein labeled with affinity-purified antifilamin. $A$ band coelectrophoresing with filamin and a band of lower molecular weight are labeled. ( $H$ ) Total myotube protein labeled with repurified antifilamin. The only labeled band coelectrophoreses with filamin. The markers next to lane $\boldsymbol{A}$ indicate the following apparent polypeptide chain molecular weights (in kilodaltons from top to bottom): 206, 116, 100, 92, 68, 42.

\section{RESULTS}

The experiments described here were designed to localize actin and actinassociated proteins within the AChR clusters of cultured rat myotubes by fluorescence techniques. For these experiments to be successful, the reagents used to label the cytoskeleton must be reliable and specific, and the methods that expose the cytoskeleton must not alter intracellular organization.

Most of the antibodies we used were affinitiy-purified rabbit antibodies generated to proteins purified from chicken gizzard, which have been partially characterized elsewhere [5]. We studied their specificity for rat myotube proteins in "Western" nitrocellulose blots. The results (Fig. 1) showed that each affinitypurified antibody we used reacted specifically with a polypeptide of the same molecular weight as the protein antigen. With the exception of antifilamin, the affinity-purified antibodies showed little cross-reaction with other proteins in extracts of rat myotubes. Antifilamin reacted with polypeptides smaller than intact filamin $\left(M_{\mathrm{r}} 250,000\right.$ : Fig. 1, lane $\left.G\right)$. We therefore purified the antibody further by adsorbing it to nitrocellulose blots of the intact polypeptide and eluting 
it with acid [32]. The repurified antibody no longer reacted with smaller polypeptides in blots of intact rat myotubes (Fig. 1, lane $H$ ), and so we used this preparation for subsequent immunofluorescence experiments. To localize actin, we used both fluorescent phalloidin derivatives, whose specificity has been wellestablished (e.g., Refs. [33, 34]), and a monoclonal antibody to actin (HP249). We have previously shown by immunoblotting that this antibody is specific for actin in extracts of rat myotubes [4].

The localization of proteins within cells by immunofluorescence requires that the cell membrane be ruptured, but this process alone could cause significant redistribution of membrane-bound cytoskeletal proteins. To minimize the possibility that our methods affected our results, we used two very different procedures to permeabilize cells and to observe the distribution of cytoskeletal proteins at AChR clusters. Both procedures were made possible by the observation that the AChR clusters of cultured rat myotubes form preferentially at sites of myotube-substrate attachment. In one set of experiments, cells were permeabilized with detergent, fixed, labeled with antibodies and with fluoresceinated antiantibodies, and then observed by TIRF. This method leaves the bulk of the myotube intact but specifically visualizes fluorescence label within $\sim 0.2 \mu \mathrm{m}$ of the glass substrate [18]. Alternatively, cells were sheared with a stream of buffer, fixed, labeled with primary and secondary antibodies, and observed by standard epifluorescence. This procedure removes the bulk of the myoplasm and contaminating proteins, leaving AChR clusters on the coverslip exposed to the bathing solution. The shearing procedure has already been used to establish the preferential association of the 43K protein with AChR domains [35], and of vinculin with the contact domains within AChR clusters [16]. TIRF micrographs of immunofluorescence due to antivinculin and anti-43K protein, shown in Fig. 2, confirm our earlier reports with sheared clusters. We therefore used both techniques to investigate the other proteins of interest in this study, actin, filamin, and $\alpha$ actinin. Talin was investigated using only samples prepared by shearing.

We found that the talin, $a$-actinin, and filamin were associated preferentially with the contact domains of AChR clusters (Fig. 3). As in the case of vinculin (Figs. $2 C$ and $D$ ), talin usually appeared in long, tapering lines coincident with AChR-poor regions within or, more frequently, at the edges of AChR clusters (Figs. $3 A$ and $B$ ). Filamin was present in a similar pattern, whether viewed in sheared samples or by TIRF (Figs. $3 C-F$ ). In experiments to localize $\alpha$-actinin, we occasionally found anti- $\alpha$-actinin immunofluorescence distributed within sheared AChR clusters in linear regions poor in AChR (Figs. $3 I$ and $J$ ). More frequently, however, this antibody yielded a punctate labeling pattern (e.g., Figs. $3 G$ and $H$ ). We do not understand the reasons for this variability. These results are consistent with the idea that, like vinculin, talin, filamin, and $\alpha$-actinin are enriched at the contact domains of AChR clusters.

Vinculin, talin, and $\alpha$-actinin have been localized in mononucleate cells where bundles of microfilaments associate with the cell membrane. We used antibodies to actin and fluorescent phalloidin derivatives to learn if similar structures were present where these proteins were enriched in AChR clusters. Sheared AChR 

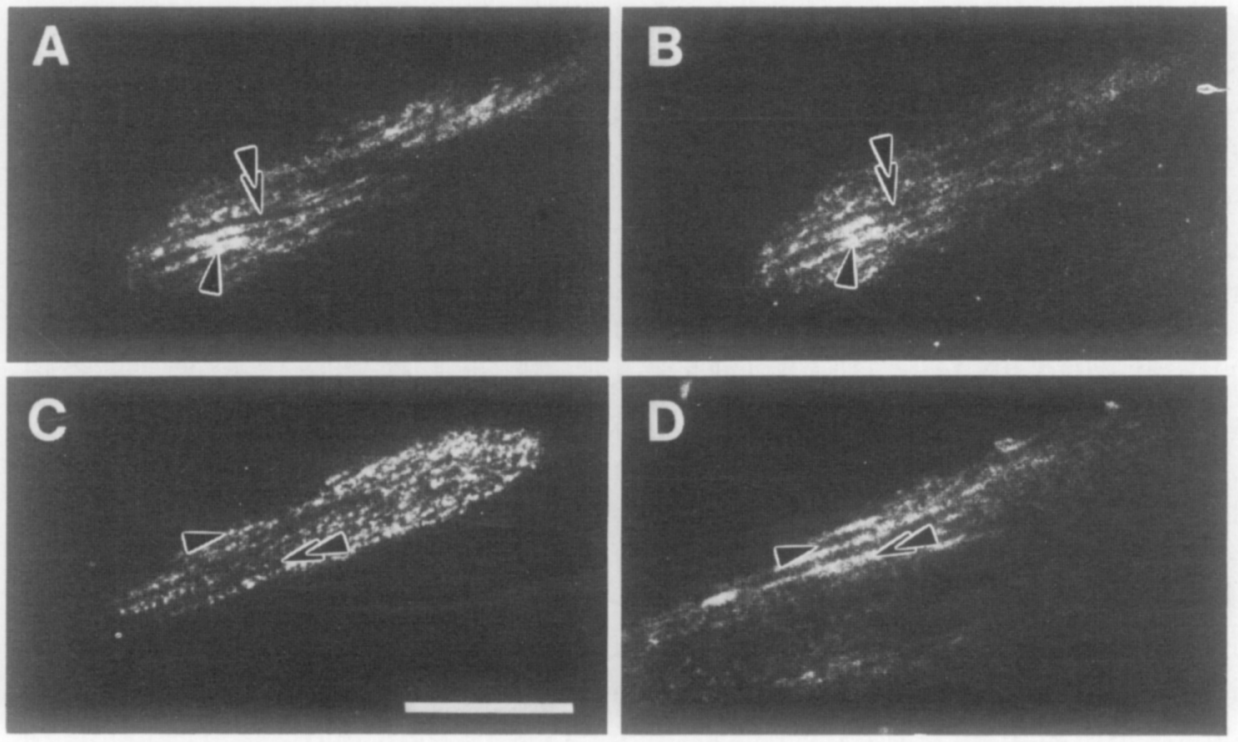

Fig. 2. Visualization of vinculin and 43K protein at AChR clusters by TIRF. Rat myotube cultures were labeled with F-BT, extracted briefly with $0.5 \%$ Triton X-100, fixed, and counterstained with monoclonal antibodies to the $43 \mathrm{~K}$ protein followed by RGAM, or with affinity-purified rabbit antivinculin, followed by RGAR. Samples were mounted in a small chamber and purged of oxygen enzymatically (see Methods). They were observed and photographed by TIRF. Antibody distribution is shown in $(B)$ and $(D)$; F-BT distribution is shown in $(A)$ and $(C)$. For this and the following figures, the long axis of the cell is parallel to the lines that are usually apparent within AChR clusters. The width of the cell is usually slightly greater than the width of the cluster itself. ( $A$ and $B$ ) Labeling for 43K protein shows that the antibody preferentially labels the AChR domains (arrowheads), not the contact domains (double arrowheads). ( $C$ and $D$ ) Labeling for vinculin shows that the antibody preferentially labels the contact domains (double arrowheads), not AChR domains (arrowheads). Bar, $20 \mu \mathrm{m}$.

clusters labeled with fluoresceinated phalloidin showed phalloidin to be concentrated in long lines overlying membrane areas poor in $\mathrm{AChR}$, but not over the AChR-rich domains themselves (Figs. $4 C$ and $D$, arrowheads). In agreement with this observation, AChR clusters isolated by extraction with saponin [36], which display actin at AChR domains but have no recognizable structures at contact domains $[4,36$ ], also fail to label with phalloidin (Figs. $4 G$ and $H$ ).

In contrast to our results with phalloidin, antibodies to actin consistently labeled both $\mathrm{AChR}$ and contact domains (Figs. $4 A, B, E$ and $F$ ). In sheared samples (Figs. $4 A$ and $B$ ), actin labeling of contact domains and $A C h R$ domains appears discrete, with brighter "darts" of immunofluorescence label overlying contact domains, and a more uniform "meshwork" overlying AChR domains. In samples examined by TIRF (Figs. $4 E$ and $F$ ), antiactin usually labels receptorrich and receptor-poor domains equally well (e.g., Figs. $4 E$ and $F$, arrows). Occasionally, however, antibody labeling was bright at AChR-rich domains and $\operatorname{dim}$ at AChR-poor regions (Figs. $4 E$ and $F$, arrowheads and double arrowheads). These results suggest that actin is present throughout the AChR cluster, where it 

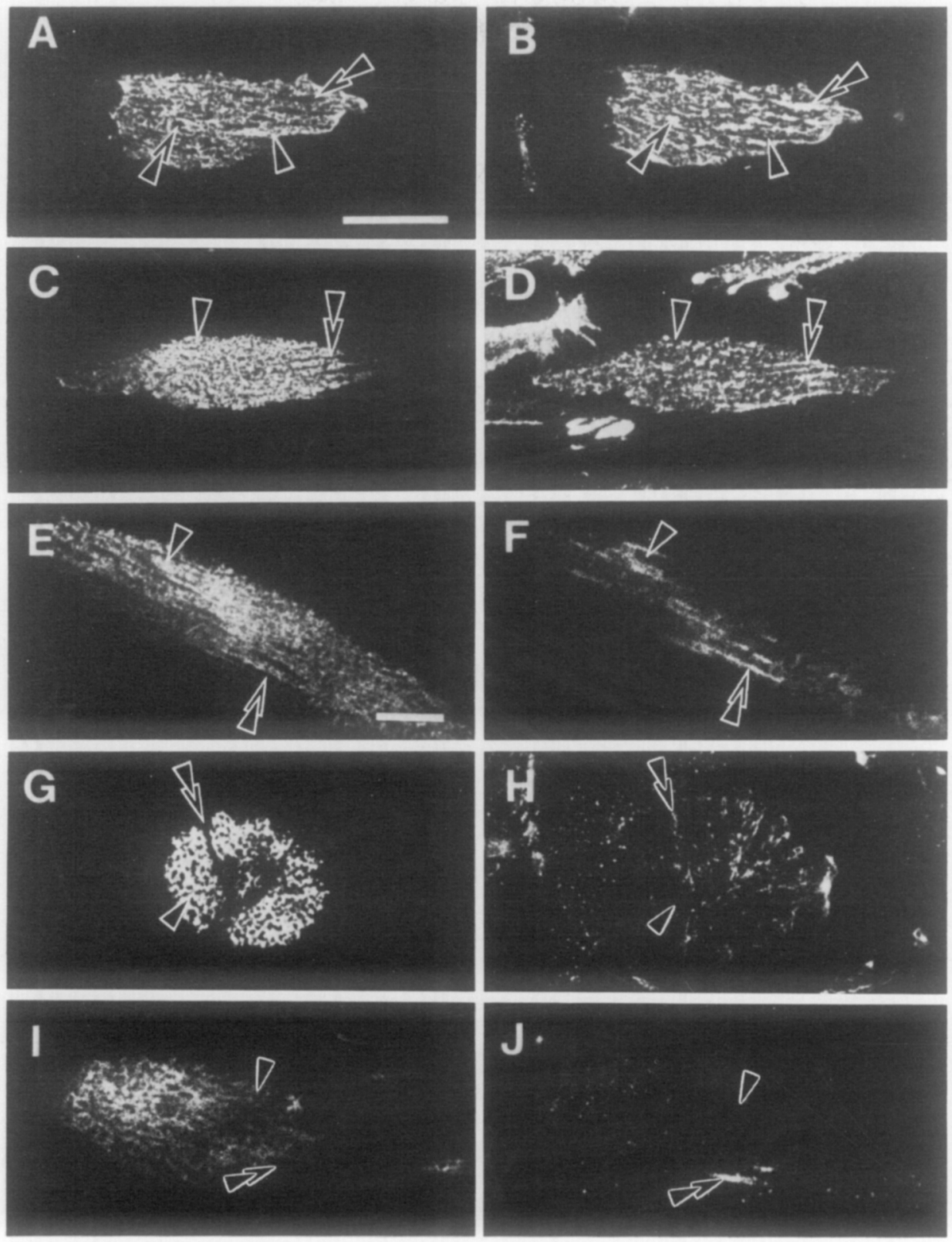

Fig. 3. Labeling of AChR clusters with antitalin, antifilamin, and anti- $\alpha$-actinin, observed after shearing or by TIRF. Rat myotubes were labeled with R-BT or F-BT, and then either sheared or permeabilized with Triton, and fixed and labeled for immunofluorescence. $(A, C, E, G$ and $I)$ AChR distribution revealed with fluorescent BT derivatives; $(B, D, F, H$ and $J)$ Distribution of cytoskeletal proteins revealed by antibodies. In all cases, antibody label concentrates at the contact domains (double arrowheads) but not the AChR domains (arrowheads) of $\mathrm{AChR}$ clusters. ( $A$ and $B$ ) Sheared AChR cluster labeled with R-BT, and antitalin followed by FGAR. ( $C$ and $D$ ) Sheared AChR cluster labeled with R-BT, and repurified antifilamin followed by FGAR, ( $E$ and $F$ ) Triton-permeabilized cluster labeled with F-BT and antifilamin, followed by RGAR, and observed by TIRF. ( $G$ and $H$ ) Sheared AChR cluster labeled with R-BT and anti-a-actinin, followed by FGAR. ( $I$ and $J$ ) Tritonpermeabilized cell labeled with F-BT and anti- $\alpha$-actinin, followed by RGAR, and observed by TIRF. Bar in $(A)$ represents $10 \mu \mathrm{m}$ and applies to panels $(A-D)$, and $(G$ and $H)$; bar in $(E)$ applies to panels $(E, F, I$, and $J$, and represents $10 \mu \mathrm{m}$. 

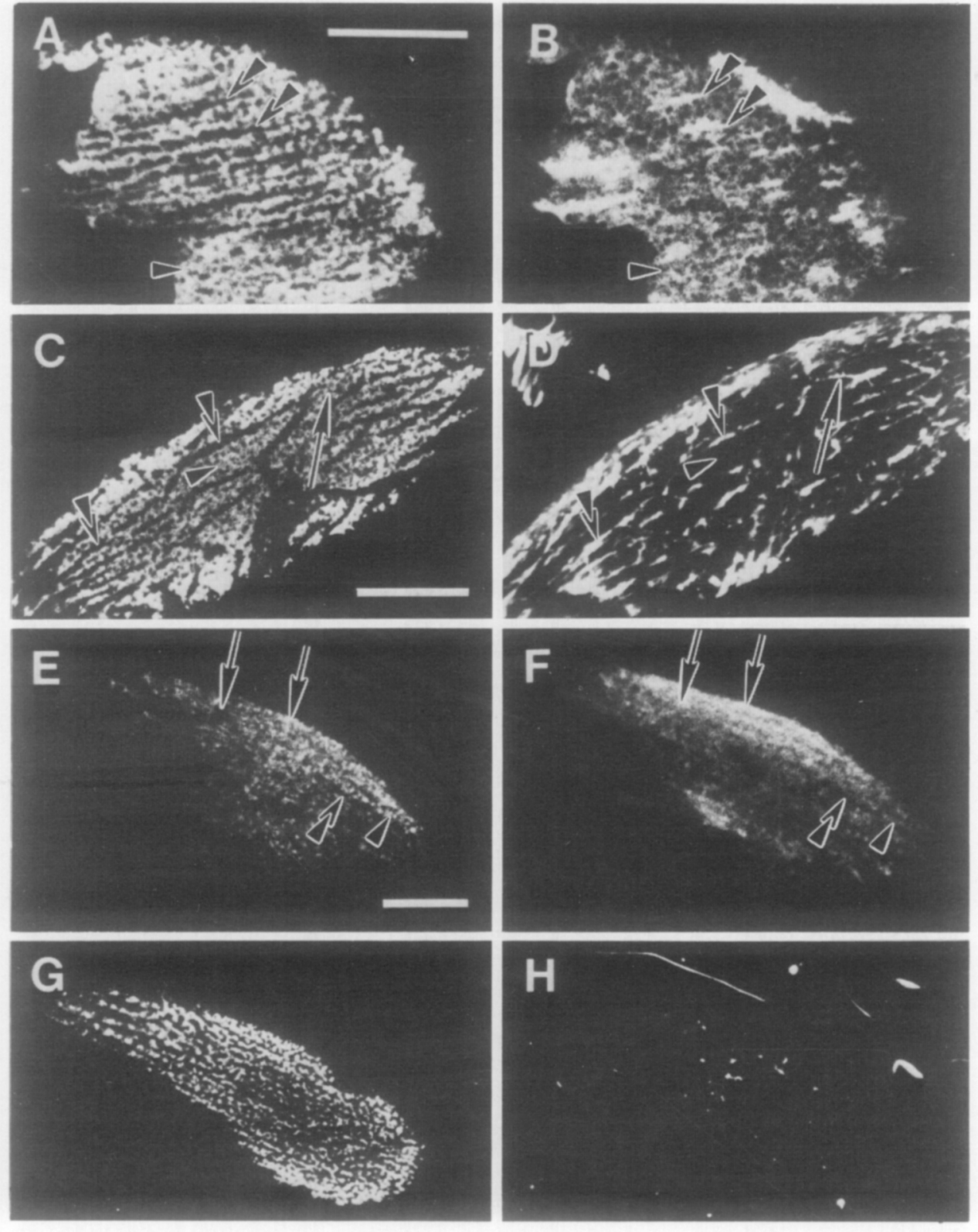

Fig. 4. Actin at AChR clusters observed by TIRF or after shearing. Rat myotubes were labeled with R-BT or F-BT, and then either sheared or permeabilized with Triton, and fixed and labeled for actin using fluoresceinated phalloidin $(D$ and $H$ ) or HP249 antiactin followed by FGAM $(B$ and $F$ ). Panels $(A, C, E$, an $\mathcal{B} G)$ show the distribution of $\mathrm{AChR}$, as revealed by fluorescent $\mathrm{BT}$ derivatives. Panels $(B$, $D, F$, and $H$ ) show the distribution of actin, as revealed by antiactin or by phalloidin. Contact domains (double arrowheads) and AChR domains (arrowheads) are indicated. ( $A$ and $B$ ) Sheared cluster labeled with R-BT and HP249 antiactin, followed by FGAM. Both contact domains and AChR domains are labeled by the antibody. $(C$ and $D$ ) Sheared cluster labeled with R-BT and fluoresceinphalloidin. Coitact domains are preferentially labeled by phalloidin (e.g., double arrowheads), but some brightly labeled structures overlie AChR domains (arrows). These can usually be ascribed to bundles of microfilaments that pass over $\mathrm{AChR}$ domains without attaching to the membrane, and that are thus in a different plane of focus. ( $E$ and $F$ ) Triton-permeabilized cell labeled with F-BT and 

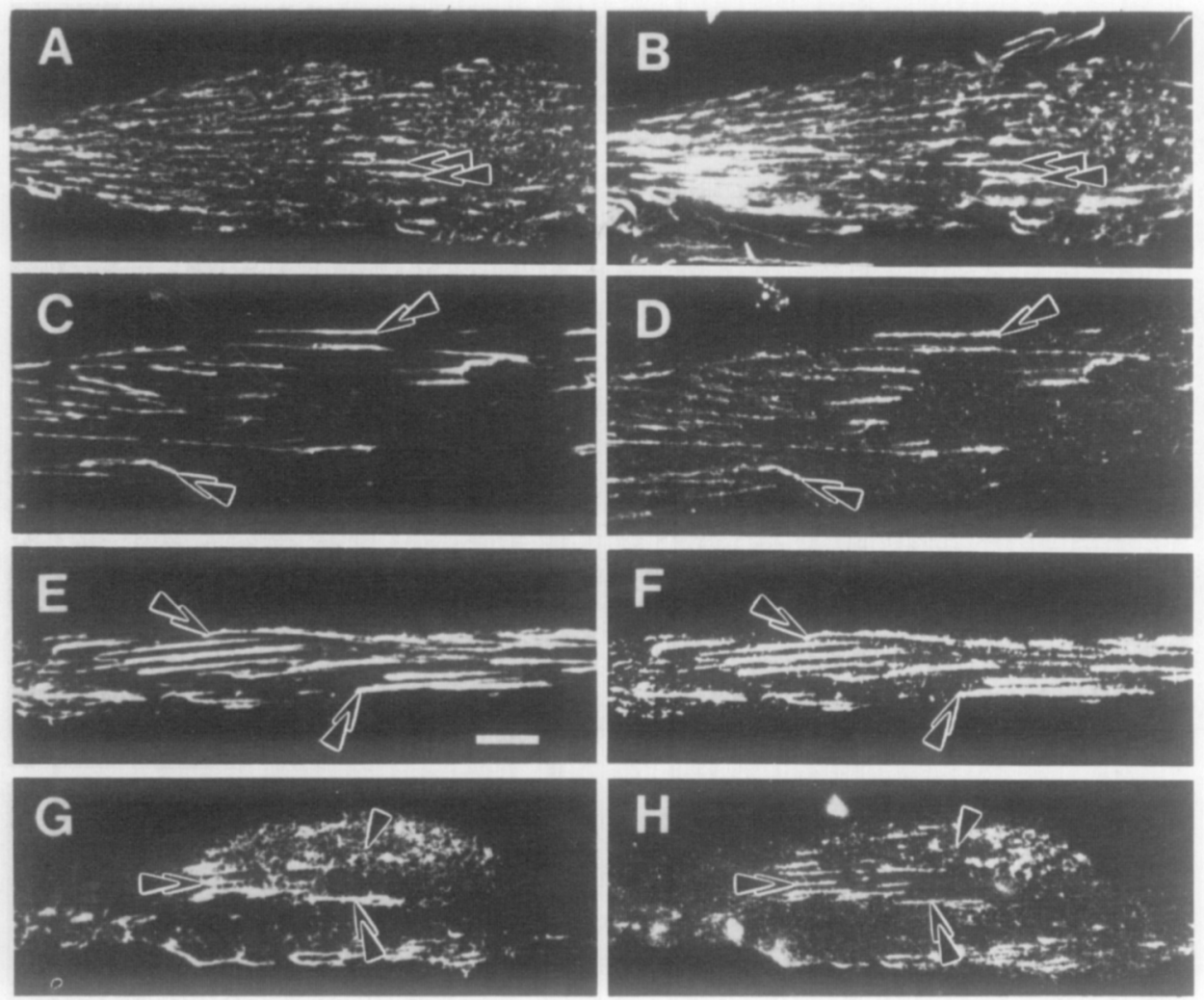

Fig. 5. Coincidence of structures labeled by antibodies and by phalloidin. Rat myotubes were sheared, fixed and labeled with antibodies, followed by FGAR, and then counterstained with rhodaminylated phalloidin (R-Ph). One sample ( $G$ and $H$ ) was prelabeled with R-BT. Panels $(A, C, E$, and $G$ ) show label from R-Ph, and in the case of panel $(G)$, from both R-Ph and R-BT. Panels $(B, D, F$, and $H$ ) show labeling by antibodies and FGAR. ( $A$ and $B$ ) Antivinculin. ( $C$ and $D$ ) Antitalin. $(E$ and $F$ ) Anti- $\alpha$-actinin. All three antibodies label structures that are also labeled by $\mathrm{R}-\mathrm{Ph}$ (double arrowheads). ( $G$ and $H$ ) Antivinculin labels structures within AChR clusters that are also labeled by $\mathrm{R}-\mathrm{Ph}$ (double arrowheads), but not by R-BT (arrowheads). Note that in panel (G), R-Ph does not label the AChR domains to any significant extent, and thus does not overlap labeling by R-BT (cf., Fig. $4 C$ and $D)$. The bar in $(E)$ represents $10 \mu \mathrm{m}$ and applies to all panels.

is recognized by antiactin antibodies. To account for the failure of phalloidin to label AChR domains, we postulate that the actin present at AChR domains either does not react well with phalloidin derivatives or is not present at concentrations high enough to be observed using these probes.

We performed double labeling experiments with antibodies and phalloidin to

HP249 antiactin, followed by RGAM and observed by TIRF. On the right, AChR domains are labeled preferentially (single and double arrowheads). On the left, both AChR-rich and AChR-poor domains are brightly labeled (arrows). ( $G$ and $H$ ) Saponin isolated AChR cluster labeled with R-BT and fluorescein-phalloidin. Phalloidin label is absent from clusters treated with saponin, but AChR-rich domains are clearly labeled with R-BT. Bars are for each pair of micrographs, except bar in $(C)$, which applies to panels $(C, D, G$, and $H$ ). Each bar represents $10 \mu \mathrm{m}$. 
determine if vinculin, talin, $\alpha$-actinin, and filamin coincided with microfilament bundles. In the first series of experiments, sheared clusters were labeled first with antibodies and FGAR, and then with R-phalloidin ( $R-P h)$. As pointed out above, phalloidin preferentially labels the actin at contact domains. The bright linear staining by the antivinculin, antitalin, and anti- $\alpha$-actinin always corresponded to sites of bright phallodin labeling (Figs. $5 A-F$ ). Similar results were obtained with antifilamin (not shown). We then repeated these experiments using samples that had been prelabeled with R-BT, to enable us to detect AChR clusters. These samples, labeled with both R-BT and R-phalloidin, showed clear AChR clusters marked with bright "darts" of phalloidin label (Fig. $5 G$, double arrowheads), present at the contact domains of the clusters (e.g., Figs. $4 C$ and $D$ ). Labeling with antivinculin was coincident with these "darts" of R-Ph label (Fig. $5 \mathrm{H}$, double arrowheads) but not with the dimmer, more uniform labeling for AChR (arrowheads). Similar results were obtained with antitalin, antifilamin, and, in some samples, anti- $\alpha$-actinin (not shown). Thus, these cytoskeletal proteins are localized within AChR clusters to receptor-poor regions that also stain with phalloidin.

\section{DISCUSSION}

The postsynaptic membrane of the vertebrate neuromuscular junction is underlain by a rich, interweaving network of cytoplasmic filaments [37, 38], that may help to maintain its distinctive structure. Presumably, these filaments interact with the postsynaptic membrane and the synaptic basal lamina to generate and stabilize the synaptic folds and the paracrystalline arrays of AChR characteristic of the adult synapse. The way they do this is unknown. To understand this process, we must identify the various proteins that are enriched postsynaptically and learn how they interact with each other and with the membrane.

We have used rat muscle cells in tissue culture as a model system in which these interactions can be more readily studied. The AChR clusters of these cells form preferentially at sites of myotube-substrate attachment. Such substrateassociated clusters remain attached to the coverslip after shearing, allowing the cytoskeletal proteins to be studied in the virtual absence of cytoplasmic contamination. Similarly, TIRF microscopy relies on the closeness of the cluster to the glass coverslip, and visualizes only those structures lying within $\sim 0.2 \mu \mathrm{m}$ of the AChR-rich membrane. Both methods suffer from the disadvantage that shearing or detergent permeabilization may dislodge or reorient the proteins associated with clustered AChR. In fact, although actin is usually present at both AChR-rich and AChR-poor domains, our results suggest that actin may occasionally be destabilized during sample preparation, as antiactin labeling of some AChR-poor domains is sometimes hard to detect (e.g., Figs. $4 E$ and $F$, double arrowheads). This observation confirms a previous report that contact domains within AChR clusters of detergent-permeabilized myotubes occasionally fail to label with antivinculin [16]. Despite this reservation, we consider it unlikely that the two, very dissimilar, methods we have used would consistently cause the same sets of 
changes in AChR clusters. In addition, the distribution of proteins in clusters prepared by shearing with paraformaldehyde (earlier experiments in this study) was identical to that of proteins at clusters that were pretreated with $\mathrm{ZnCl}_{2}$ before shearing and fixation. As the distribution of all the proteins we studied using TIRF was consistent with that we obtained using sheared samples, we conclude that the localization of these proteins was probably not artifactual.

Although these two techniques yielded the same results, a comparison of TIRF with labeling after shearing suggests that the latter procedure has two advantages. First, of course, it is performed with simple equipment and requires only a microscope equipped for epifluorescence; a laser is not needed. Second, the quality of the images obtained with sheared samples is generally better than that obtained with TIRF. This is probably due to several factors. (i) Permeabilization of the cells with detergent, required for TIRF, causes the organization of AChR clusters to appear less distinct. This was observed in earlier studies of the 43K protein at AChR clusters [35]. (ii) TIRF detects the fluorescence arising from regions up to $0.2 \mu \mathrm{m}$ into the cytoplasm. This may introduce a background signal that is higher than that obtained after shearing, which can yield AChR clusters with essentially no cytoplasmic contamination [39]. (iii) Illumination in TIRF tends to be less uniform than illumination by a mercury arc, due to interference fringes formed by the coherent laser beam. All of these factors contribute to the higher clarity of images obtained with sheared samples. Shearing and subsequent immunolabeling are therefore the easier method for studying the cytoskeleton in fixed samples of AChR clusters of rat myotubes. On the other hand, TIRF can be used on living cells, and so is the method of choice for studies requiring sequential observations of $\mathrm{AChR}$ and associated proteins.

Our results show that vinculin, talin, $\alpha$-actinin, and filamin are highly enriched on the cytoplasmic surface of the AChR-poor contact domains of AChR clusters. Of course, we cannot exclude the possibility that these four proteins are present at much lower densities at AChR-rich domains, and are simply undetected by our methods. These same proteins are also enriched at the focal contacts of cultured mononucleate cells [12-15], where the cell membrane comes within $10 \mathrm{~nm}$ of the substrate [40]. Focal contacts are also the sites where bundles of actin microfilaments (stress fibers) attach to the cell membrane (e.g., [41, 42]). Our observations with fluorescent phalloidin derivatives suggest that similar microfilament bundles are associated with the contact domains of rat myotubes. Pumplin has reached a similar conclusion using ultrastructural techniques [39]. These results suggest great similarity between contact domains of $\mathrm{AChR}$ clusters in myotubes and focal contacts of other cells. They support the idea that a large portion of the membrane of $\mathrm{AChR}$ clusters in vitro is devoted to stabilizing the attachment of actin filaments to the membrane, and thereby to extracellular structures. Vinculin in adult chicken muscle may play a comparable role, stabilizing the attachment of intracellular structures to the membrane and to the extracellular matrix [43]. The attachment of chick myotubes to extracellular structures in culture is presumably also stabilized by similar structures, which are enriched in vinculin, CSAT (a fibronectin receptor), and actin [44]. It is not yet known if vinculin and the other 
cytoskeletal proteins of contact domains fulfill a similar function at the postsynaptic membrane of the neuromuscular junction.

Although bundles of microfilaments are clearly enriched at contact domains, actin is also present over the AChR domains of both sheared and Triton X-100permeabilized AChR clusters. This result agrees with a previous report, which showed that actin was present in the AChR domains of clusters isolated using saponin, and that its selective removal caused AChR to redistribute in the cluster membrane [4]. The actin in AChR domains seems to be visible reliably only with antiactin antibodies, however, and is not easily seen with fluorescent phalloidins. Phalloidin also fails to label AChR clusters isolated with saponin, which also lack vinculin, $\alpha$-actinin, filamin, and talin [36; R. J. Bloch, unpublished], but contain intact AChR domains [36]. The ability of phalloidin to label the actin at contact but not AChR domains may be due in part to the fact that bundles of actin filaments are considerably easier to visualize with phalloidin than are individual actin filaments. If, as has been suggested [11, 36, 45], actin at $\mathrm{AChR}$ domains is organized as short oligomers like the actin in the membrane skeleton of the human erythrocyte [46], then one might expect very little labeling by phalloidin. Recent experiments further suggest that, like the human erythrocyte membrane, AChR domains (but not the contact domains) contain $\beta$-spectrin [47].

Our results are therefore consistent with the idea that actin is organized in two distinct structures within AChR clusters, and that these are associated with different cytoskeletal proteins. The actin at contact domains is organized as bundles of microfilaments and is associated with vinculin, talin, $\alpha$-actinin, and filamin. The actin at AChR domains may be filamentous, but it most probably is not present as bundles of long filaments, and, together with spectrin [47], it is associated more directly with AChR and the AChR-linked 43K protein. Because the $43 \mathrm{~K}$ protein is closely associated with $\mathrm{AChR}$, some investigators have suggested that it may mediate the binding of $\mathrm{AChR}$ at the postsynaptic membrane to underlying cytoskeletal proteins (reviewed in [10,11]). There is some evidence that the 43K protein can bind to actin [48]. Thus, the actin at AChR domains may interact indirectly with $\mathrm{AChR}$, and so participate in the formation or stabilization of AChR clusters.

Although we have discussed two domains in AChR clusters-the contact and AChR domains-our evidence suggests that there are other regions that are poor both in AChR and in actin and actin-associated proteins. These regions are especially apparent in Fig. $5 G$. In that experiment, AChR domains and contact domains were stained with R-BT and R-phalloidin, but the cluster still showed distinct patches of membrane that remained unlabeled. Such patches were not regions that retained vinculin, talin, or the other proteins of the contact domain, but had lost microfilament bundles, because no labeling with any of our antibodies could be detected there. Although some of these areas may have been generated by excessive shearing (see above), we believe that most of them constitute a third distinct domain within AChR clusters. Recent results suggest that this third domain is composed of large plaques of coated membrane rich in clathrin [39; D. W. Pumplin, J. Strong, and R. J. Bloch, in preparation]. These 
three domains appear to account for most of the intracellular membrane surface of AChR clusters that is visible in the light microscope. We are therefore close to defining the molecular composition of the different cytoskeletal domains associated with AChR clusters in vitro. The challenge is now to relate these structures to synaptogenesis in vivo.

We thank Wendy Resneck for her expert assistance in many of these experiments, Beth Concaugh and Yvette Dorsey for preparing the cultures, Drs. J. Wilkins, K. Burridge, J. Fee, and S. Froehner for their generous gifts of reagents, and Drs. D. Pumplin and P. Luther for their comments on earlier versions of this paper. Our research has been supported by grants from the National Institutes of Health to R.J.B. (NS 17282 and NS 22652) and to D.A. (NS 14565), and by a grant to R.J.B. from the Muscular Dystrophy Association. M.V. was the recipient of an International Fellowship from the American Association of University Women. R.J.B. was the recipient of a Research Career Development Award (NS 00679).

\section{REFERENCES}

1. Fambrough, D. M. (1979) Physiol. Rev. 59, 165.

2. Salpeter, M. M. (1987) The Vertebrate Neuromuscular Junction (Salpeter, M. M., Ed.), p. 1, A. R. Liss, New York.

3. Hall, Z. W., Lubit, B. W., and Schwartz, J. H. (1981) J. Cell Biol. 90, 789.

4. Bloch, R. J. (1986) J. Cell Biol. 102, 1447.

5. Bloch, R. J., and Hall, Z. W. (1983) J. Cell Biol. 97, 217.

6. Sealock, R., Paschal, B., Beckerle, M., and Burridge, K. (1984) Exp. Cell Res. 163, 143.

7. Burden, S. (1982) J. Cell Biol. 94, 521.

8. Froehner, S. C., Murnane, A. A., Tobler, M., Peng, H. B., and Sealock, R. (1987) J. Cell Biol. $104,1633$.

9. Woodruff, M. L., Theriot, J., and Burden, S. J. (1987) J. Cell Biol. 104, 939.

10. Froehner, S. C. (1986) Trends Neurosci. 9, 37.

11. Bloch, R. J., and Pumplin, D. W. (1988) Amer. J. Physiol. 254, C345.

12. Geiger, B. (1979) Cell 18, 193.

13. Burridge, K., and Connell, L. (1983) J. Cell Biol. 97, 359.

14. Lazarides, E., and Burridge, K. (1975) Cell 6, 289.

15. Burridge, K., and Feramisco, J. R. (1980) Cell 19, 587.

16. Bloch, R. J., and Geiger, B. (1981) Cell 21, 25.

17. Land, B. R., Podleski, T. R., Salpeter, E. E., and Salpeter, M. M. (1977) J. Physiol. $269,155$.

18. Axelrod, D. (1981) J. Cell Biol. 89, 141.

19. Moody-Corbett, F., and Cohen, M. W. (1982) J. Embryol. Exp. Morphol. 72, 53.

20. Bridgman, P. C., and Nakajima, Y. (1983) J. Cell Biol. 96, 363.

21. Pumplin, D. W., and Bloch, R. J. (1983) J. Cell Biol. 97, 1043.

22. Bloch, R. J. (1979) J. Cell Biol. 82, 626.

23. Axelrod, D. (1980) Proc. Natl. Acad. Sci. USA 77, 4823.

24. Ravdin, P., and Axelrod, D. (1977) Anal. Biochem. 80, 585; Erratum 83, 336.

25. Johnson, G. D., Davidson, R. S., McNamee, K. C., Russell, G., Goodwin, P., and Holborow, E. J. (1982) J. Immunol. Methods 55, 231.

26. Avnur, Z., and Geiger, B. (1981) J. Mol. Biol. 153, 361.

27. Aggeler, J., and Werb, Z. (1982) J. Cell Biol. 94, 613.

28. Johnson, G. D., and Nogeuira Araujo, G. M. de C. (1981) J. Immunol. Methods 43, 349.

29. Burnett, W. N. (1981) Anal. Biochem. 112, 195.

30. Laemmli, U. K. (1970) Nature (London) $227,680$.

31. Feramisco, J. R., and Burridge, K. (1980) J. Biol. Chem. 255, 1194.

32. Smith, D. E., and Fisher, P. A. (1984) J. Cell Biol. 98, 20.

33. Wulf, E., Deboben, A., Bautz, F. A., Faulstich, H., and Wieland, T. (1979) Proc. Natl. Acad. Sci. USA 76, 4498.

34. Barak, L. S., Yocum, R. R., Nothnagel, E. A., and Webb, W. W. (1980) Proc. Natl. Acad. Sci. USA 77, 980.

35. Bloch, R. J., and Froehner, S. C. (1987) J. Cell Biol. 104, 645. 
36. Bloch, R. J. (1984) J. Cell Biol. 99, 984.

37. Ellisman, M. H., Rash, J. E., Staehelin, A., and Porter, K. R. (1976) J. Cell Biol. 68, 752.

38. Hirokawa, N., and Heuser, J. E. (1982) J. Neurocytol. 11, 487.

39. Pumplin, D. W. (1987) J. Cell Biol. 105, 61 a.

40. Maupin, P., and Pollard, T. D. (1983) J. Cell Biol. 96, 51.

41. Heath, J. P., and Dunn, G. A. (1978) J. Cell Sci. 29, 197.

42. Wehland, J., Osborn, M., and Weber, K. (1979) J. Cell Sci. 37, 257.

43. Shear, C. R., and Bloch, R. J. (1985) J. Cell Biol. 101, 240.

44. Damsky, C. H., Knudsen, K. A., Bradley, D., Buck, C. A., and Horwitz, A. F. (1985) J. Cell Biol. 100, 1528.

45. Pumplin, D. W., and Bloch, R. J. (1987) J. Cell Biol. 104, 97.

46. Pinder, J. C., and Gratzer, W. B. (1983) J. Cell Biol. 96, 768.

47. Bloch, R. J., and Morrow, J. S. (1989) J. Cell Biol. 108, 481.

48. Walker, J. H., Boustead, C. M., and Witzemann, V. (1984) EMBO J. 3, 2287.

Received February 2, 1989 